\title{
Menschenwürde: unterschiedliche Auslegungen, unterschiedliche Auswirkungen
}

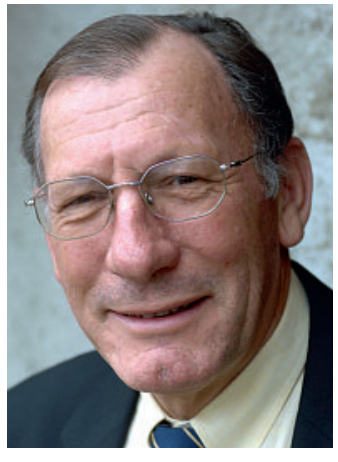

Jean Martin
Die Nationale Ethikkommission hat kürzlich eine Sitzung dem Thema «Kultur des Sterbens» gewidmet. Philosophen kamen dabei ebenso zu Wort wie Vertreter der Gesundheitsberufe. In den Beiträgen war viel von Menschenwürde die Rede. Angelsächsische Ethiker und Wissenschaftler wie Ruth Macklin, Peter Singer oder Steven Pinker stellen den Begriff zwar als nutzlos (useless concept) in Frage, doch in der Allgemeinen Erklärung der Menschenrechte sowie in der europäischen Ethik und Rechtslehre spielt er weiterhin eine grundlegende Rolle. Und er ist mit einer ausgeprägten intuitiven Auffassung verknüpft. In unseren Diskussionen wurde darauf hingewiesen, dass das Wort Würde in unterschiedlicher Bedeutung verwendet wird.

Man kann Würde im Sinne von Anständigkeit auffassen, von Nicht-Verfall oder besser von Selbstachtung - als Weigerung, ein Leben zu führen, das dessen beraubt wurde, was ihm Würde verliehen hat. Eine zweite Auslegung, die oft mit der ersten zusammenhängt, sieht die Würde im Sinne von Selbstbestimmung, in Verbindung mit dem Recht des Einzelnen, frei darüber zu entscheiden, was er mit seinem Leben anfangen will. Man darf annehmen, dass diese Lesart von den drei Vierteln der Schweizer geteilt wird, die laut wiederholter Umfragen wollen, dass Sterbehilfe eine Option ist (auch wenn nur eine sehr kleine Minderheit diese in Anspruch nehmen würde). Der Begriff ist vielleicht wenig präzise, doch sie verstehen ihn intuitiv und brauchen keine theoretischen Ausführungen (wie ich auch in einer nordamerikanischen Debatte glaubte beitragen zu können [1]). Ein eher induktiver als deduktiver Ansatz.

Etwas ganz anderes ist die ontologische Auffassung, die sich auf die Essenz des Daseins bezieht; ein metaphysischer Begriff - die Würde wird dem Menschen gegeben und ist eine unverrückbare Grösse, unabhängig von jeder anderen Überlegung. Ich möchte hier in aller Bescheidenheit einwenden, dass ich die Würde des Menschen eher für etwas halte, das allmählich entstanden ist, auf unterschiedlichen Wegen in den verschiedenen Gesellschaften (und die geschichtliche Entwicklung der Ereignisse und Ideen scheint mir hier recht zu geben), und nicht für etwas Naturgegebenes. Dieses menschliche Konstrukt muss ständig verteidigt und weiter verbessert werden - das ist unsere Verantwortung. Vielleicht ist auch der Begriff Achtung angebrachter: Wir müssen jedem Menschen, so wie er ist, konstant und bewusst Achtung entgegenbringen. «Würde ist zunächst einmal die logische Voraussetzung, Achtung schuldig zu sein (...) Indem sie die Verantwortung übernimmt, eine Welt der Achtung herbeizuführen, lebt die Menschheit ihre eigene Würde.» [2].
Diejenigen, die von einer ontologisch gegebenen Würde ausgehen, sind im Sinne dieser metaphysischen Essenz gegen den Gedanken der Freiheit des Einzelnen, über sein Leben zu bestimmen. In der Praxis bedeutet das tendenziell die Befürwortung umfassender medizinischer Massnahmen am Ende des Lebens in einer Form, die andere eher als Verbohrtheit bezeichnen würden (Verlängerung des Lebens oder des Sterbens?). Diejenigen, die Würde als Ablehnung des Verfalls und als Ausübung ihrer Selbstbestimmtheit auffassen, werden hingegen solche «heldenhaften» Bemühungen logischerweise ablehnen und den Abbruch von Massnahmen verlangen, die sie als sinnlos erachten.

Natürlich gilt der Grundsatz, dass jeder für sich die Auslegung von Würde wählen kann, die seinem Wertesystem entspricht. Es geht in keiner Weise darum, hier Vorschriften zu machen. Es sollte jedoch zum Nachdenken anregen, zu welchen unterschiedlichen praktischen Auswirkungen die beiden Denkweisen führen, unter anderem auch aus wirtschaftlicher Sicht. Ich möchte unterstreichen, dass ich die Vorbehalte all der Ärzte, Pflegepersonen und Bürger teile, die es alarmierend finden, wenn ökonomische Zwänge die Qualität oder allgemeine Verfügbarkeit der medizinischen Versorgung gefährden, doch ich glaube auch, dass wir (im Rahmen der Res publica) vor der wirtschaftlichen und politischen Realität nicht die Augen verschliessen können. Dass die Werte, die sich aus den Rechten des Einzelnen und aus der Berufsethik ableiten, unbedingt schützenswert sind, dass jedem Menschen unabhängig von seiner Lebenslage die gleiche Achtung gebührt - all das ändert nichts daran, dass Mittel, die für den einen Zweck aufgewendet wurden, für einen anderen nicht mehr verfügbar sind. Und es ist ganz offensichtlich nicht mehr vorstellbar - es sei denn, in der Gesellschaft vollzöge sich ein Gesinnungswandel, für den ich aber keine Anzeichen sehe -, dem Bereich der medizinischen Versorgung seine monetären Mittel zu entziehen.

In der notwendigen Diskussion zum Thema Menschenwürde darf nicht verschwiegen werden, dass man je nach vertretener Position die nach wie vor begrenzten Mittel unterschiedlich zwischen den Patienten, die unterschiedliche Leistungen brauchen, aufteilt. Das könnte in nicht unerheblichem Masse diskriminierende Folgen im Hinblick auf einen optimalen Zugang zu Versorgungsleistungen nach sich ziehen.

Dr. Jean Martin, Mitglied der Redaktion und der nationalen Ethikkommission

(Der Autor äussert hier seine persönliche Meinung.) 